\title{
Multiple stellar populations in massive LMC star clusters
}

\author{
A. D. Mackey, P. Broby Nielsen, A. M. N. Ferguson \\ and J. C. Richardson
}

Institute for Astronomy, University of Edinburgh, Royal Observatory, Blackford Hill, Edinburgh, EH9 3HJ, UK, email: dmy@roe.ac.uk

\begin{abstract}
The recent discovery of multiple stellar populations in massive Galactic globular clusters poses a serious challenge for models of star cluster formation and evolution. A new angle on this problem is being provided by rich intermediate-age clusters in the Magellanic Clouds. In this contribution we describe the discovery of three such LMC clusters with peculiar main-sequence turn-off morphologies. The simplest interpretation of our observations is that each of these three clusters is comprised of two or more stellar populations spanning an age interval of $\sim 300$ Myr. Surprisingly, such features may not be unusual in this type of cluster.
\end{abstract}

Keywords. globular clusters: general, galaxies: individual (LMC), Magellanic Clouds, galaxies: star clusters

\section{Introduction}

It is a long-held astrophysical tenet that rich star clusters, including globular clusters, are composed of single stellar populations. In many cases there is an abundance of excellent supporting evidence - see, for example, the spectacular colour-magnitude diagrams (CMDs) presented recently for NGC 6397 by Richer et al. (2008).

New observations, however, are challenging this accepted picture. It has recently been discovered that several of the most massive Galactic globular clusters harbour multiple stellar populations with a wide variety of unexpected characteristics (e.g., Piotto et al. 2008). The most strikingly unusual CMD belongs to $\omega$ Centauri, which exhibits at least four different populations covering a wide spread in metal abundance and age across the sub-giant branch (SGB) (Villanova et al. 2007), as well as a main sequence bifurcation which can only be explained by the presence of a vastly helium-enriched population (Piotto et al. 2005). NGC 2808 possesses a triple main sequence split, again implying the presence of helium-enriched populations (Piotto et al. 2007); while NGC1851 and NGC 6388 show clear splits in their SGBs, possibly indicative of populations with ages $\sim 1$ Gyr apart, or of sizeable intra-cluster variations in chemical composition (Milone et al. 2008a; Piotto et al. 2008; Salaris et al. 2008). Even the much lower mass Galactic globular cluster M 4, which does not display a markedly unusual CMD, apparently possesses two stellar populations - one comprised of Na-rich CN-strong stars and one of $\mathrm{Na}$-poor CN-weak stars (Marino et al. 2008). Overall, these observed properties pose serious challenges for conventional models of globular cluster formation and evolution.

Rich star clusters in the Magellanic Clouds have the potential to open a new angle on this problem. These objects have masses comparable to Galactic globulars below the peak of the luminosity function, but are typically much younger and hence possess CMDs far more sensitive to internal dispersions in age, for example. In this contribution we report on the recent discovery of several rich intermediate-age LMC clusters 

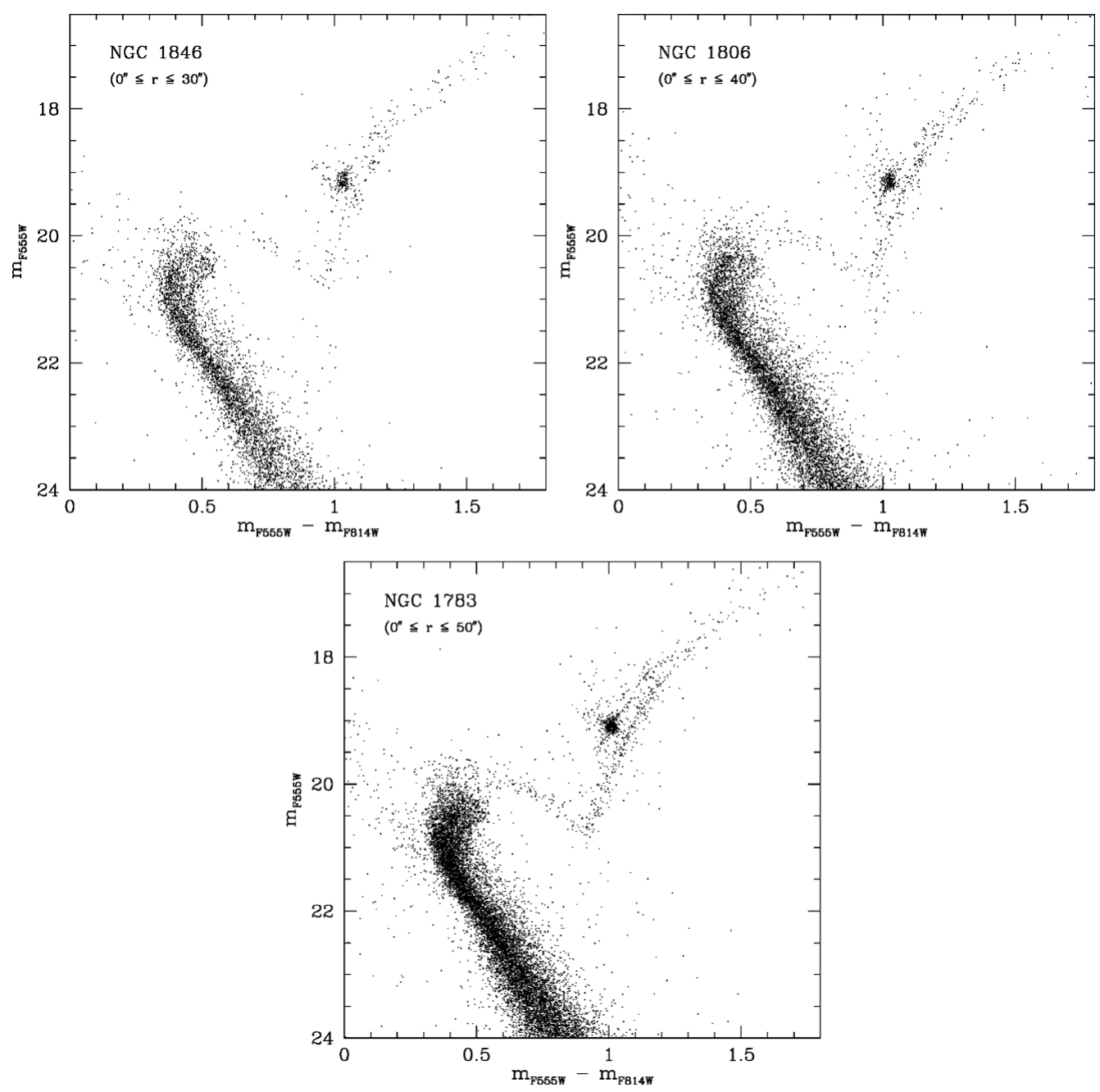

Figure 1. HST/ACS CMDs from our HST program \#9891 snapshot imaging for the three strongest candidates for possessing peculiar main-sequence turn-off morphologies in that sample. The double turn-off in NGC 1846 is clearly visible (Mackey \& Broby Nielsen 2007), while the CMD for NGC 1806 shows a hint of a double turn-off. The turn-off for NGC 1783 has no distinct branches but exhibits a rather large spread in colour. Each object possesses a narrow RGB and a compact, well-defined red clump.

with peculiar main-sequence turn-off morphologies, inconsistent with being single stellar populations.

\section{Results and analysis}

The first indication that some intermediate-age LMC clusters might possess unusual CMDs arose from data obtained with the Advanced Camera for Surveys (ACS) onboard the Hubble Space Telescope as part of our snapshot survey of rich star clusters in the Magellanic Clouds (HST Program \#9891). A careful photometric analysis revealed several objects showing unexpected features around their main-sequence turn-offs.

CMDs for the three strongest candidates in our sample - NGC 1846, 1806, and 1783 are displayed in Fig. 1. Each exhibits an unusual turn-off morphology. NGC 1846 

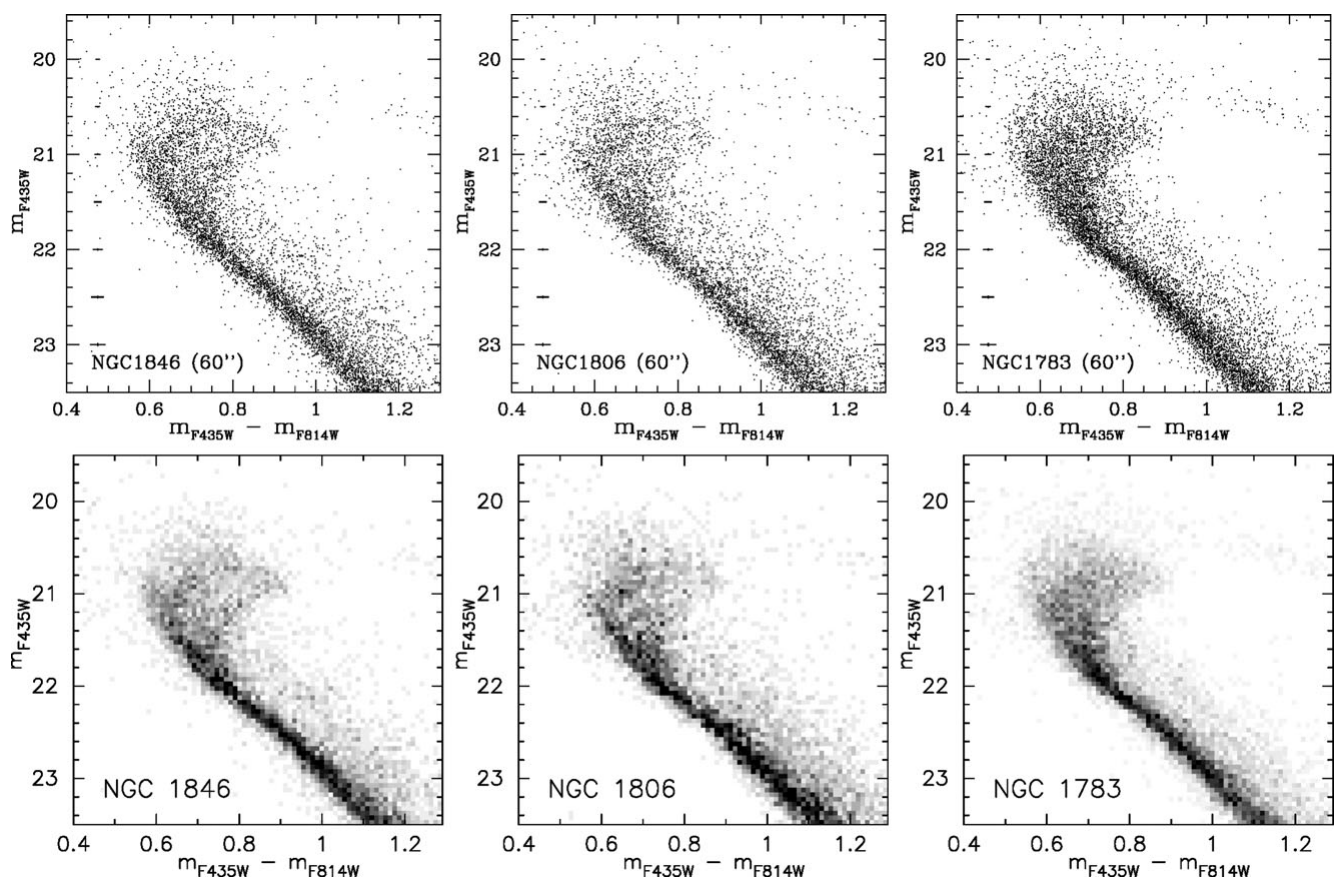

Figure 2. $H S T$ /ACS CMDs (upper panels) and Hess diagrams (lower panels) constructed from imaging taken under HST program \#10595, obtained from the public archive. These deep CMDs confirm the peculiar nature of the three clusters identified in our HST \#9891 snapshot imaging (see Fig. 1). NGC 1846 and 1806 clearly display bifurcated main-sequence turn-offs, while NGC 1783 has a turn-off exhibiting a large spread in colour. Each cluster has a narrow main sequence and a significant population of unresolved binary stars.

possesses two clear turn-off branches, while NGC1806 shows a hint of two turn-off branches. The turn-off for NGC 1783 has no distinct branches but rather exhibits a spread in colour which is larger than the observational uncertainties would suggest. Apart from these features, the CMDs are as expected for intermediate-age clusters; in particular, each object possesses a narrow RGB and a compact, well-defined red clump. A detailed analysis of the CMD for NGC 1846 is presented by Mackey \& Broby Nielsen (2007).

Motivated by our discoveries, we searched the HST public archive for additional imaging of these three clusters. All were observed with ACS as part of HST Program \#10595 between September 2005 - January 2006. These data allowed the construction of higher $\mathrm{S} / \mathrm{N}$ CMDs than was possible from our snapshot imaging. These archival CMDs are displayed in Fig. 2, and unambiguously show that both NGC 1846 and 1806 possess two main-sequence turn-off branches, while NGC 1783 possesses a turn-off covering a spread in colour much larger than can be explained by the photometric uncertainties (note the very narrow upper main sequence). This feature may represent a bifurcated turn-off in which the branches are unresolved on the CMD, or it may represent a smooth spread of stars. More information on these clusters and their CMDs is presented by Mackey et al. (2008); see also Goudfrooij et al. (2008) and Milone et al. (2008b).

Apart from the peculiar main-sequence turn-off morphologies, each cluster CMD exhibits a very narrow RGB and main sequence. This implies that the turn-off features are not due to significant line-of-sight depth or differential reddening in these clusters. The narrow sequences further suggest minimal internal dispersions in $[\mathrm{Fe} / \mathrm{H}]$ in each of the three clusters; however we note they place no constraints on the possibility of internal 

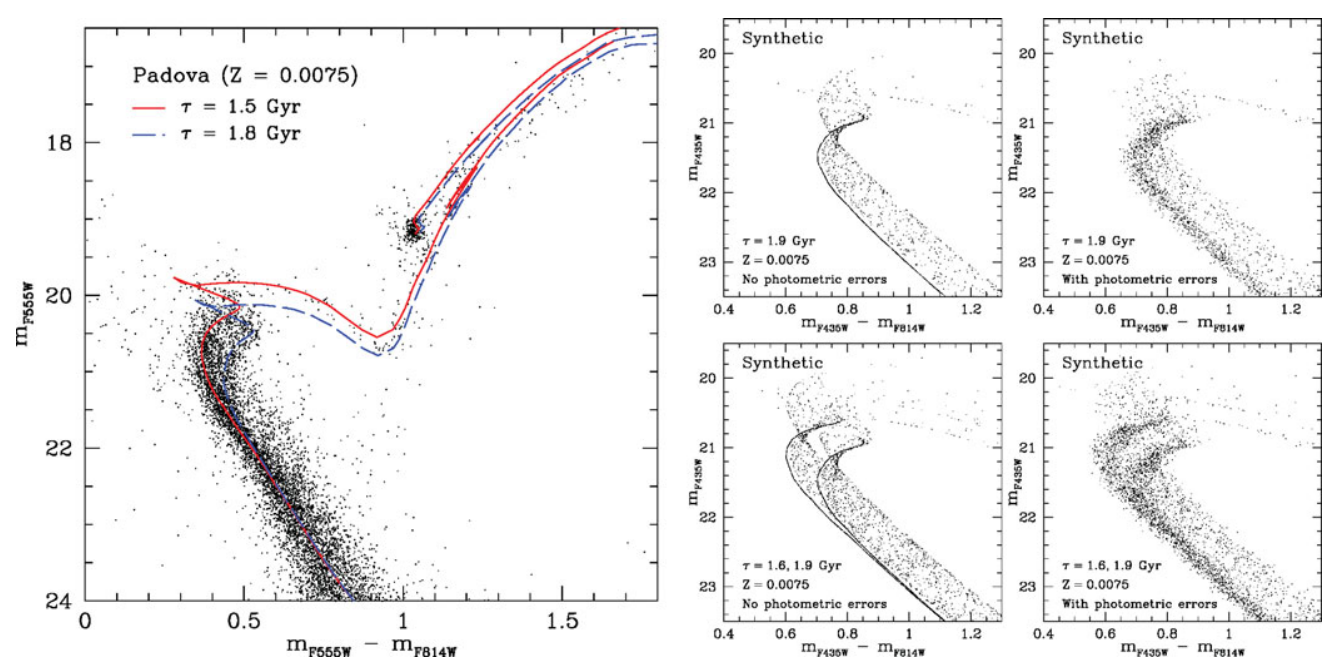

Figure 3. Left: Isochrone fit to the HST \#9891 CMD for NGC 1846. Under the assumption of chemical homogeneity and a uniform distance and foreground extinction, an age spread of $\sim 300$ Myr within this $\sim 1.8$ Gyr old cluster can closely reproduce the observed CMD.

Right: Synthetic CMDs aimed at reproducing the HST \#10595 CMD for NGC 1846. Each includes a population of $40 \%$ unresolved binaries. The upper panels include only a single stellar population, while the lower panels include two stellar populations with ages as indicated. Unresolved binaries cannot alone reproduce the observed bifurcated turn-off.

variations in other chemical abundances - for example, $\mathrm{CN}, \mathrm{O}, \mathrm{Na}$, or $[\alpha / \mathrm{Fe}]$ - as are observed for several of the peculiar massive Galactic globular clusters (e.g., Piotto et al. 2008). Mucciarelli et al. (2008b) (see also the contribution by Mucciarelli in these proceedings) obtained high resolution spectra for 6 RGB stars in NGC 1783 and found no significant star-to-star dispersion in $[\alpha / \mathrm{Fe}]$; however similar measurements are not presently available for NGC 1846 or 1806. In the absence of this information, we assume complete chemical homogeneity in each of the three systems.

In this scenario, the simplest interpretation of the observed CMDs is that each cluster possesses at least two stellar populations of differing ages. To quantify this, we fit isochrones from the Padova and BaSTI groups (Marigo et al. 2008; Pietrinferni et al. $2004)$ to the CMDs. Details of the fitting procedure may be found in Mackey \& Broby Nielsen (2007) and Mackey et al. (2008). An example may be seen in the left panel of Fig. 3, which shows two Padova isochrones with $Z=0.0075$ and ages $1.5 \mathrm{Gyr}$ and 1.8 Gyr fit to our HST \#9891 snapshot CMD for NGC 1846. These models closely reproduce the observed features of the CMD, suggesting that NGC 1846 may consist of two populations with an age difference of $\sim 300 \mathrm{Myr}$. We obtained identical results for NGC 1806 and 1783. For this latter, we fit isochrones to the upper and lower envelopes of the turn-off, to indicate the maximum expected age spread implied by this feature.

Evident on each CMD (especially in Fig. 2) is a spread of stars above and to the red of the main sequence. These are due to unresolved binaries, and imply non-negligible populations of such objects in the three clusters. In fact, in the central cluster regions, counts of stellar detections lying along the main and binary sequences suggest that the ratio of the number of unresolved binary systems with mass ratios $q \gtrsim 0.5$ to the total number of detected objects may locally be as high as $\sim 30-40 \%$. We constructed synthetic CMDs to investigate the role played by unresolved binary stars around the turn-off regions, and in particular investigate whether these objects might reproduce the observed turn-off structures. Full details may be found in Mackey et al. (2008). 

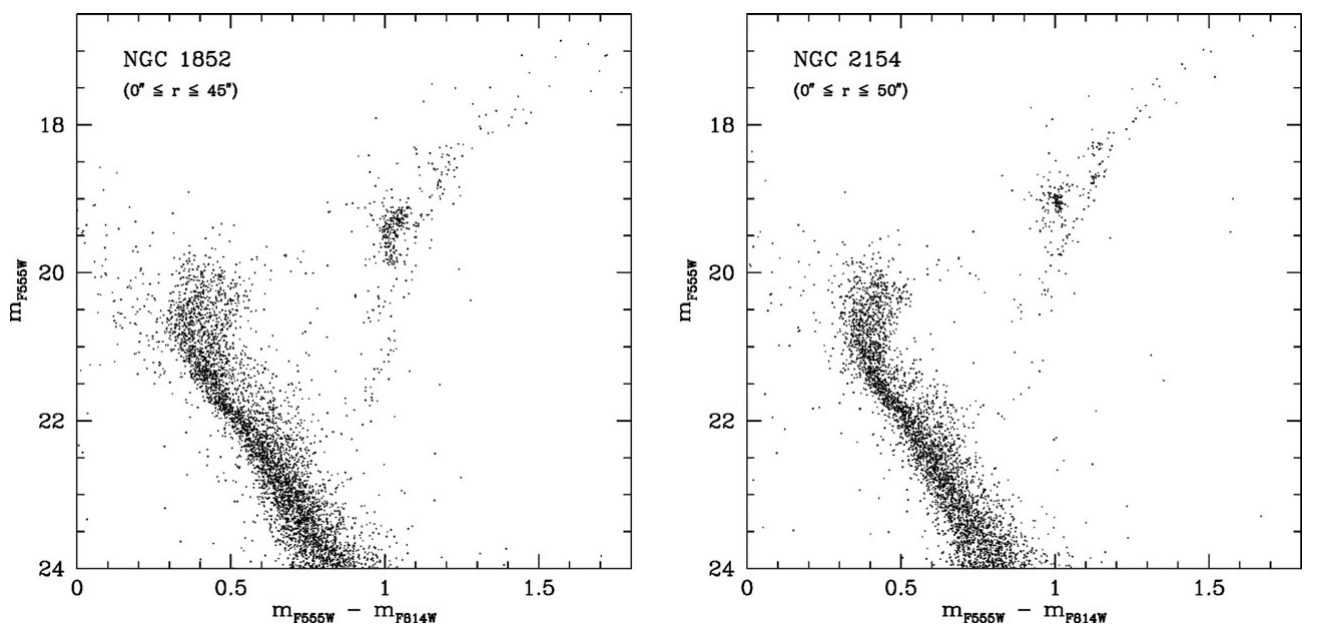

Figure 4. HST /ACS CMDs from our HST program \#9891 snapshot imaging for two additional candidates for possessing peculiar turn-off morphologies. NGC 1852 potentially exhibits a bifurcated turn-off, while NGC 2154 has a turn-off spanning a large spread in colour. Both clusters suffer from relatively strong contaminating field populations; even so, Milone et al. (2008b) have independently confirmed the unusual nature of these two objects.

Examples for NGC 1846 are shown in the right panel of Fig. 3, aimed at reproducing the observed CMD from Fig. 2. The upper panels show synthetic CMDs constructed using a single stellar population of age $1.9 \mathrm{Gyr}$ and comprised of $40 \%$ unresolved binaries with $q>0.5$. The left-hand panel shows the synthetic CMD as generated, while the righthand panel shows the same CMD with random photometric uncertainties added to each star. These uncertainties were generated to match those determined for detected stars in NGC 1846. These two plots clearly demonstrate that unresolved binaries add confusion and some intrinsic spread around the main-sequence turn-off, but cannot alone reproduce the peculiar observed structure for NGC 1846. Specifically, the binary sequence does not result in a distinct branch above and to the blue of the lower turn-off branch.

The lower two panels show a synthetic CMD constructed using two single stellar populations of ages 1.6 and 1.9 Gyr. As before, $40 \%$ unresolved binaries with $q>0.5$ were included. This new CMD closely resembles the observed CMD, demonstrating that two stellar populations in NGC 1846 (and by extension NGC 1806) are necessary to explain the observed turn-off structure. NGC 1783 may require more than two turn-offs, or two more closely spaced branches.

We conclude this section by noting that NGC 1846, 1806, and 1783 are not the only intermediate-age Magellanic Cloud clusters which have been found to possess unusual main-sequence turn-off morphologies. In our HST \#9891 data we discovered several additional candidates, the two strongest of which are shown in Fig. 4. NGC 1852 shows a potentially bifurcated turn-off, while NGC 2154 has a turn-off spanning a relatively large spread in colour. Milone et al. (2008b) have conducted a thorough analysis of archival HST data for 16 intermediate-age LMC clusters, confirming all five of our discoveries and adding an additional six. Furthermore, Glatt et al. (2008) have recently shown that the SMC cluster NGC 419 may well possess a peculiar turn-off morphology. To this sample of objects we should add the LMC cluster NGC 2173, which was shown several years ago to be likely to have a spread at the turn-off larger than expected from measured photometric uncertainties (Bertelli et al. 2003). Several younger LMC clusters, such as NGC 1868 and NGC 2011, have also been identified as possessing irregular CMDs (Santiago et al. 2002; Gouliermis et al. 2006). 


\section{Discussion}

The rapidly-growing number of intermediate-age Magellanic Cloud star clusters that are being found to possess peculiar main-sequence turn-off morphologies suggests that such a feature may not be uncommon for this type of object. This is a surprising result since Magellanic Cloud star clusters have long been treated as prototypical single stellar populations, used to test and calibrate stellar evolution models.

As discussed by Mackey \& Broby Nielsen (2007) and Mackey et al. (2008), if we are correct in interpreting the observed cluster CMDs in terms of internal age spreads, identifying a viable scenario for their formation is not trivial. It is difficult to imagine how the three LMC clusters discussed in this contribution, each at least an order of magnitude less massive than the peculiar Galactic globulars, might have retained sufficient unenriched gas to undergo multiple widely-separated episodes of star formation. One possible alternative to these suggestions is that each peculiar cluster is the merger product of two or more clusters formed separately within a single giant molecular cloud (Mackey \& Broby Nielsen 2007). The LMC possesses numerous "double" star clusters (e.g., NGC 1850) and its low tidal field could facilitate such mergers. A second alternative, raised by several participants at this conference, is that the peculiar turn-off features may not be due to more than one stellar population, but rather a hitherto poorly-appreciated aspect of the evolution of intermediate-age stars. However, this model may have trouble explaining why a significant number of intermediate-age Magellanic Cloud clusters do not exhibit unusual turn-off morphologies (e.g., Milone et al. 2008b).

Additional observations are clearly important in constraining viable formation scenarios for these objects. In particular, it is crucial to understand in detail the chemical composition of each peculiar cluster. If these objects are not chemically homogeneous then the requirement for such large internal age spreads as are currently being assumed may well be obviated. Such measurements are under way at the time of writing, and new information should shortly be available.

\section{References}

Bertelli, G., Nasi, E., Girardi, L., Chiosi, C., Zoccali, M., \& Gallart, C. 2003, AJ, 125, 770

Glatt, K., Grebel, E. K., Sabbi, E., et al. 2008, AJ, 136, 1703

Goudfrooij, P., Puzia, T. H., Kozhurina-Platais, V., \& Chandar, R. 2008, AJ, submitted

Gouliermis, D. A., Lianou, S., Kontizas, M., Kontizas, E., \& Dapergolas, A. 2006, ApJ, 652, L93

Mackey, A. D. \& Broby Nielsen, P. 2007, MNRAS, 379, 151

Mackey, A. D., Broby Nielsen, P., Ferguson, A. M. N., \& Richardson, J. C. 2008, ApJ, 681, L17

Marigo, P., Girardi, L., Bressan, A., Groenewegen, M. A. T., Silva, L., \& Granato, G. L. 2008, $A \& A, 482,883$

Marino, A. F., Villanova, S., Piotto, G., Milone, A. P., Momany, Y., Bedin, L. R., \& Medling, A. M. 2008, A\&BA, 490, 625

Milone, A. P., Bedin, L. R., Piotto, G., et al. 2008a, ApJ, 673, 241

Milone, A. P., Bedin, L. R., Piotto, G., \& Anderson, J. 2008b, $A \mathscr{E} A$, in press

Mucciarelli, A., Carretta, E., Origlia, L., \& Ferraro, F. R. 2008, AJ, 136, 375

Pietrinferni, A., Cassisi, S., Salaris, M., \& Castelli, F. 2004, ApJ, 612, 168

Piotto, G., Villanova, S., Bedin, L. R., et al. 2005, ApJ, 621, 777

Piotto, G., Bedin, L. R., Anderson, J., et al. 2007, ApJ, 661, L53

Piotto, G. 2008, MemSAI, 79, 3

Richer, H. B., Dotter, A., Hurley, J., et al. 2008, AJ, 135, 2141

Salaris, M., Cassisi, S., \& Pietrinferni, A. 2008, ApJ, 678, L25

Santiago, B., Kerber, L., Castro, R., \& de Grijs, R. 2002, MNRAS, 336, 139

Villanova, S., Piotto, G., King, I. R., et al. 2007, ApJ, 663, 296 